\title{
Image Informative Maps for Estimating Noise Standard Deviation and Texture Parameters
}

\author{
M. Uss, ${ }^{1,2}$ B. Vozel, ${ }^{1}$ V. Lukin, ${ }^{3}$ S. Abramov, ${ }^{3}$ I. Baryshev, ${ }^{2}$ and K. Chehdi ${ }^{1}$ \\ ${ }^{1}$ TSI2M Laboratory, University of Rennes 1, BP 80518, 22305 Lannion cedex, France \\ ${ }^{2}$ Department of Design of Aircraft Radio-Electronic Systems, National Aerospace University (Kharkov Aviation Institute), \\ 17 Chkalova Street, 61070 Kharkov, Ukraine \\ ${ }^{3}$ Department of Receivers, Transmitters and Signal Processing, National Aerospace University (Kharkov Aviation Institute), \\ 17 Chkalova Street, 61070 Kharkov, Ukraine
}

Correspondence should be addressed to B. Vozel, benoit.vozel@enssat.fr

Received 7 December 2010; Accepted 21 February 2011

Academic Editor: Joao Manuel R. S. Tavares

Copyright (C) 2011 M. Uss et al. This is an open access article distributed under the Creative Commons Attribution License, which permits unrestricted use, distribution, and reproduction in any medium, provided the original work is properly cited.

\begin{abstract}
The problem of automatic detection of image areas appropriate for accurate estimation of additive noise standard deviation (STD) irrespectively to processed image properties is considered in this paper. For accurate estimation of either image texture or noise STD, we distinguish two complementary informative maps: noise- (NI-) and texture- (TI-) informative ones. The NI map is determined and iteratively upgraded based on the Fisher information on noise STD calculated in scanning window (SW) fashion. Fractional Brownian motion ( $\mathrm{fBm}$ ) model for image texture is used to derive the required Fisher information. To extract final noise STD from NI map, fBm- and DCT-based estimators are implemented. The performance of these two estimators is comparatively assessed on large image database for different noise levels. It is also compared with performance of two competitive state-of-the-art estimators recently published. Utilizing NI map along with DCT-based noise STD estimator has proved to be significantly more efficient.
\end{abstract}

\section{Introduction}

Images formed by multispectral sensors or digital cameras are subject to undesirable errors including sensor random noise, blur, distortions, radiometrical, and geometrical errors. These effects are to be detected and quantified, either prior to subsequent image processing, aiming at their compensation (filtering or deblurring) or prior to image low level information extracting [1]. Sensor random noise is often a dominant factor degrading image quality [1-3]. Although there are methods that are able to perform image processing (segmentation, denoising, edge detection, classification, etc.) without using a priori knowledge or pre-estimating noise STD (segmentation methods $[4,5]$ are interesting examples of such methods), better performance is usually provided for techniques that exploit such a priori information or estimation of noise STD [6]. For example, BM3D filer, one of the best filters available today [7], requires noise STD to be known or pre-estimated [8].
It is often desirable to estimate noise STD in a blind manner. First of all, blind processing allows dealing with a large amount of data acquired by modern sensors. Second, potentially, performance of blind methods is higher than that of a human operator, as they may use subtle difference between image content and noise that can be not visible for a human eye. To reach good performance in practice, a blind estimator of noise STD should satisfy the following requirements [2]: (1) to provide unbiased estimates with variance as small as possible; (2) to perform well enough at different noise levels; (3) to be not sensitive to image content, that is, to provide appropriate accuracy even for textural images. These requirements are controversial and it has been found a difficult problem to satisfy them altogether.

The problem of blind estimation of sensor random noise STD has been extensively studied by the research community for the last two decades (see [2] and references therein). It has been mainly stressed that not all local areas in an observed image are suitable for providing accurate estimates of noise 
STD. Indeed, it is preferable to use those areas where signal and noise can be quite easily separated. The easiest situation for benefiting from such separation is maybe in the spatial domain. Spatial methods make essential use of the processed image areas with negligible level of texture spatial variation compared to the noise level, so-called homogeneous areas (HA) [3]. As HAs hypothesis could be quite restrictive due to a limited area of HAs within processed images (authors of the paper [9] reported $0.6-2 \%$ of HAs in Visible InfraRed Scanner, VIRS-200, hyperspectral image), spatial methods may fail to provide practically desired accuracy, and may result in biased noise STD estimates [10] (see results of testing method [11] by Roger and Arnold in [12]). To get around these shortcomings, methods operating in the spectral domain utilize the fact that image texture is known to be usually smoother than noise. As a result, after applying suitable decorrelation transform, texture is concentrated in low frequency transform coefficients in contrast to the noise which spreads uniformly among low and high transform coefficients (for spatially uncorrelated noise). DCT [13], 3D DCT [14], and wavelet $[15,16]$ transforms have been selected for this purpose in the past.

Typically, positions of HAs or nonintensive texture areas are not known in advance [9]. And using nonappropriate textural areas (even nonintensive ones) for noise STD estimation may lead to outliers that can degrade the final noise STD estimate. Performance reduction of both spatial and spectral state-of the-art estimators for textural images [10] is mainly explained by aforesaid reason. Two different ways to cope with this problem have been proposed so far. The first one is to consider the whole image for noise STD estimation and to reject outliers that come from textural areas by a robust postprocessing of local estimates [9]. The main idea consists in splitting an image into small fragments, calculating for them local means and local STDs $[11,17]$. Then the final noise STD can be estimated either by robustly fitting linear model to the corresponding scatter plot [18] or by robust finding the histogram mode of local variance estimates $[11,19]$. However, this approach fails to provide accurate estimates if HAs and areas with nonintensive texture cover less than $10-20 \%$ of the image [20].

The second approach is to consider complementary preclassification to preliminary determine suitable areas (either HAs or nonintensive texture areas) [21]. In paper [22], HAs are selected interactively and manually for hyperspectral images. But this task is not so easy for large and complex images and can lead to erroneous selections. Besides it is labor consuming and requires qualified operators. Automatic classification of the processed image into textural and homogeneous areas [23] or the Automated Local Convergence Locator (ALCL) proposed in [24] can be considered for both facilitating and improving HAs selection accuracy [23]. When the obtained classification is effective enough, these processing stages can contribute to improve the performance of a noise STD estimation method as it was shown for method [19] for textural images [25].

However, classification methods may also fail to discriminate correctly between areas appropriate and nonappropriate for noise STD estimation, because of the textured features of the processed image and/or the noise level. In this case, the discrimination errors lead to outliers and to performance degradation of the whole procedure, including classification and noise STD estimation itself. As a result, preclassification and robust postprocessing stages are to be used simultaneously [21].

This paper concentrates on the problem of blind estimation of sensor random noise STD from noisy textured images. The noise is assumed additive signal-independent and uncorrelated (i.i.d). Although this model is slightly idealized, it is widely used for images formed by old generation hyperspectral sensors, color images, and so forth [26]. In this paper, we propose and describe a new estimation scheme that belongs to the preclassification approach mentioned above. Our main goal is to demonstrate the proposed approach ability to significantly improve noise STD estimation accuracy over the state of the art. That is why we have restricted ourselves to additive noise case for which sophisticated estimators have been proposed and simulation results are available in the literature. Note that our approach is not limited to the additive noise case; the same formalism can be followed for signal-dependent noise as well as for correlated noise.

In our approach, we mainly deal with textural images and texture parameters are estimated as well but only as a support for the main problem of noise STD estimation. The approach novelty is that it presumes finding image NI and TI areas where information is understood in Fisher's statistical sense. All detected TI and NI areas compose, respectively, the two complementary TI and NI maps, further used to discriminate suitable SW for estimating either noise or texture parameters. For each image SW, we calculate Fisher's information (or Cramér-Rao Lower Bound, CRLB) on noise STD. Then, the SWs are sorted according to their decreasing level of information on noise STD (increasing CRLB). All SWs with information above a threshold compose NI map and the rest of them compose TI map. Thus, each SW that belongs to NI map can provide a noise STD estimate with predefined accuracy making this map especially useful for contributing to final noise STD estimation. The proposed CRLB-based approach also allows estimating potential accuracy of noise STD estimates for any single SW and for the whole NI map as well.

In contrast to some classification (or segmentation) methods where the goal is to classify image content well enough independently (under some conditions) on unknown noise level $[4,5]$, our objective, given any noise level, is not to classify image content, but simply to obtain two complementary maps. One map is intended for accurate noise estimation, the other one; for accurate image texture model estimation. In our application, for different noise levels, different NI and TI maps will be (and should be) obtained.

The main contribution of this paper consists in obtaining NI map, as defined above, directly from the noisy image. The problem is that for each SW the proposed CRLB-based criterion to decide whether this SW belongs to the NI map 
is a function of texture and noise parameters that are both unknown. To be effective, this CRLB-based criterion needs to be accurately estimated from a noisy image. The problem is that it is usually difficult to estimate texture parameters from a noisy image on one hand and noise parameters from textural image on the other hand. Indeed, starting with an initial guess for the NI and TI maps, we can expect ourselves to accurately estimate texture parameters from TI map by fixing noise STD (optimistically to a value close to the true one) and noise STD from NI map by fixing texture parameters (optimistically to a vector value close to the true one). But we can hardly expect to accurately estimate both of them from any SW in the whole image. To solve this practical issue, we propose to predict unknown texture parameters for NI map and noise STD for TI map by values estimated from the respective complementary maps. By doing this, we hope to obtain quite accurate estimates of texture and noise parameters available for any SW in the whole image. Based on this knowledge, both informative maps can be in turn refined and upgraded by considering CRLB-based criterion once again. These two stages are iterated for refining texture parameters and noise STD estimates as well as respective informative maps until convergence criterion is reached.

In practice, our method follows a nonlocal approach with respect to involved parameters. In this, it differs from the approach in paper [14] where the same nonlocal approach is used with respect to similar texture patches, as this is basically exploited in image filtering [27].

We consider 2D fBm-model for describing image texture with the Hurst exponent, $H$, translating texture roughness (correlation structure). We assume that in a neighborhood of an image pixel, texture Hurst exponent (roughness) varies only a little. On the contrary, texture amplitude can vary significantly (change in light conditions is an example), and it is possible to find both TI and NI SWs within image local neighborhood. This assumption, though simple (e.g., it does not take into account image edges), allows developing maximum likelihood (ML) noise STD estimator and confirming the practical interest of using TI and NI maps as additional sources of useful information on both texture and noise local parameters.

It is important to note that noise STD estimation is emphasized in the proposed scheme, though texture parameters (roughness and amplitude) according to the fBm model are also estimated for the whole image. These estimates can be useful for quantifying and classifying image textures [28].

This paper is organized as follows. Section 2 introduces the $\mathrm{fBm}$-field model and details of the proposed scheme based on NI and TI maps to improve texture and noise parameters estimation accuracy. We introduce Fisher information on noise STD in a single SW and explain how it is utilized for building NI and TI maps. Specific estimators are defined for texture parameters and noise STD in, respectively, NI and TI maps. In Section 3, we comparatively assess the proposed noise STD estimators on large database of reallife images with other state-of-the-art estimators. Finally, in Section 4, we conclude the work.

\section{Noise STD Estimation Based on Texture- and Noise-Informative Maps}

2.1. The Proposed Approach. We denote by $y(t, s)$ an observed image for which we assume additive noise model

$$
y(t, s)=x(t, s)+n(t, s)
$$

where $x(t, s)$ is the corresponding noise-free image at pixel position $(t, s)$ and $n(t, s)$ is a normally distributed spatially uncorrelated random field with zero mean and variance $\sigma_{n}^{2}$.

When solving the problem of blind noise STD estimation in an $N \times N$ SW fashion, we would like to determine which SWs should be used for noise STD estimation and which should be rejected. Let us discuss this task from the Fisher's information point of view. We assign a Fisher's information for noise STD estimation $I_{\text {STD }}$ to each SW. By setting a threshold on $I_{\mathrm{STD}}$, it is possible to divide all image SWs into two groups: (1) SWs with $I_{\text {STD }}$ above the threshold and (2) SWs with $I_{\text {STD }}$ below the threshold. The first group of SWs corresponds to HAs or areas with nonintensive texture that allow to accurately estimate noise STD, provided texture parameters are known. Thus, we call them NI SWs. The second group of SWs corresponds to textural areas. Such areas are noninformative for noise STD estimation, but they are able to provide helpful and quite accurate information on image texture parameters. Thus we call them TI SWs. All NI and TI SWs compose NI and TI maps, respectively.

Only those SWs that belong to the NI map should be basically used for noise STD estimation (see discussion below in Section 2.3). The use of SWs from the TI map would lead to overbiased estimates due to strong outliers [2]. As image areas that correspond to NI and TI maps are not known in advance, finding NI SWs is a crucial primary task for blind noise parameters estimation. To solve this problem, $I_{\mathrm{STD}}$ should be estimated in SWs fashion directly from noisy image. Note that Fisher information is a function of both texture and noise true parameters which are all unknown. Unfortunately, these parameters cannot be accurately estimated from a single SW: a given informative map can only provide accurate estimates either for texture parameters or noise STD.

To overcome this difficulty, we propose on one hand, to predict texture parameters in NI map with parameters estimated from neighboring TI map. On the other hand, noise STD in TI map is to be estimated from NI map (see Figure 1). By making use of such an alternative estimation scheme, it seems possible to estimate quite accurately both noise and texture parameters for the whole image allowing reliable Fisher information calculation and further discriminating between NI/TI SWs.

In the proposed scheme, the estimation of either texture parameters or noise STD depends on both informative maps. The calculation of Fisher information and the determination of both informative maps, in turn, depend on texture parameters and noise STD. An iterative algorithm is to be used which successively refines texture and noise parameters estimates and both informative maps until convergence is reached (convergence is reached when noise STD estimates 


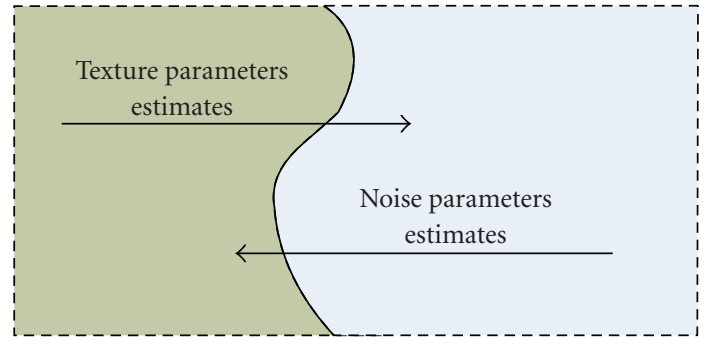

Texture-informative areas

Noise-informative areas

FIgure 1: General idea of the proposed noise STD estimation scheme.

do not change significantly between two successive iterations).

To implement the proposed alternative scheme, we need (1) to introduce suitable parametrical model for image texture; (2) to define the corresponding Fisher information (or CRLB) for noise STD estimation from a single SW and to discriminate between TI/NI SWs; (3) to implement ML estimators for texture parameters in a TI SW (fixing noise STD) and for noise STD in a NI SW (fixing texture parameters); (4) to predict texture parameters and noise STD for SWs that belong to the complementary maps based on obtained ML estimates.

2.2. Fractional Brownian Motion Model for Image Texture. We introduce $\mathrm{fBm}$ model for image texture in an $N \times N$ SW centered at $\left(t_{0}, s_{0}\right): x(t, s)=B_{H}(t, s)+m_{\left(t_{0}, s_{0}\right)}, t=$ $t_{0}-N_{h}, \ldots, t_{0}+N_{h}, s=s_{0}-N_{h}, \ldots, s_{0}+N_{h}$, where $B_{H}(t, s)$ is $2 \mathrm{D} \mathrm{fBm}$ field, $m_{\left(t_{0}, s_{0}\right)}$ is a mean bias, $N_{h}$ is a half size of the SW, $N=2 N_{h}+1$. This model has been successfully used for texture description, analysis and classification [29].

By definition $[30,31], B_{H}(t, s)$ is a nonstationary isotropic Gaussian process with origin at the point $(0,0)$, $B_{H}(0,0)=0$, with correlation function given by

$$
\begin{aligned}
& \left\langle B_{H}(t, s) \cdot B_{H}\left(t_{1}, s_{1}\right)\right\rangle \\
& =0.5 \sigma_{x}^{2}\left({\sqrt{t^{2}+s^{2}}}^{2 H}+{\sqrt{t_{1}^{2}+s_{1}^{2}}}^{2 H}-{\sqrt{\left(t-t_{1}\right)^{2}+\left(s-s_{1}\right)^{2}}}^{2 H}\right),
\end{aligned}
$$

where $H \in(0,1)$ is the Hurst exponent describing $\mathrm{fBm}$ texture roughness: $H \rightarrow 0$ for rough texture, $H \rightarrow 1$ for smooth one, $\sigma_{x}^{2}$ is the variance of increment of $\mathrm{fBm}$ process on unit distance that describes $\mathrm{fBm}$ amplitude.

2.3. CRLB on Noise STD in a Single $S W$. We define an $N_{Y} \times 1$, $N_{\mathrm{Y}}=N^{2}$, sample vector $\mathbf{Y}$ (vectors and matrices are in bold throughout the paper) consisting of pixels within $N \times N$ SW centered at $\left(t_{0}, s_{0}\right)$. As we do not know the original coordinates $(t, s)$ of $\mathrm{fBm}$ process, we should translate them to the point $\left(t_{0}, s_{0}\right)$. This can be done by transformation $\Delta B_{H}(t, s)=B_{H}(t, s)-B_{H}\left(t_{0}, s_{0}\right)$ (that eliminates unknown $\left.m_{\left(t_{0}, s_{0}\right)}\right)$ or as $\Delta \mathbf{Y}=\mathbf{Y}-y\left(t_{0}, s_{0}\right) \mathbf{1}$ where $\mathbf{1}$ is an $N_{\Delta \mathbf{Y}} \times$
1 unitary vector. In this case, the central element $y\left(t_{0}, s_{0}\right)$ should be removed from $\mathbf{Y}$ leading to $N_{\Delta \mathbf{Y}} \times 1, N_{\Delta \mathbf{Y}}=N^{2}-1$ sample $\Delta \mathbf{Y}$. The logarithmic likelihood function (LF) for the sample $\Delta \mathbf{Y}$ (omitting a constant) is given by

$$
\ln L(\Delta \mathbf{Y} ; \boldsymbol{\theta})=-\frac{1}{2}\left[\Delta \mathbf{Y}^{T} \mathbf{R}_{\Delta \mathbf{Y}}^{-1} \Delta \mathbf{Y}+\ln \left|\mathbf{R}_{\Delta \mathbf{Y}}\right|\right],
$$

where $\boldsymbol{\theta}=\left(\sigma_{x}, H, \sigma_{n}\right)$ is the $\mathrm{fBm}$-field parameter vector to be estimated, $\mathbf{R}_{\Delta \mathbf{Y}}=0.5 \sigma_{x}^{2} \mathbf{R}_{H}+\sigma_{n}^{2}(\mathbf{I}+\mathbf{J})$ is the $N_{\Delta \mathbf{Y}} \times N_{\Delta \mathbf{Y}}$ correlation matrix of $\Delta \mathbf{Y}$ defined by (2) and (1), I is $N_{\Delta \mathbf{Y}} \times$ $N_{\Delta \mathbf{Y}}$ identity matrix, $\mathbf{J}$ is $N_{\Delta \mathbf{Y}} \times N_{\Delta \mathbf{Y}}$ unit matrix, $\mathbf{R}_{H}$ is the $N_{\Delta \mathbf{Y}} \times N_{\Delta \mathbf{Y}}$ correlation matrix of unknown noiseless sample $\Delta \mathbf{X}$ normalized by the factor $\sqrt{2} / \sigma_{x}$. True values of the model parameters are denoted as $\boldsymbol{\theta}_{0}=\left(\sigma_{x 0}, H_{0}, \sigma_{n 0}\right)$.

The Fisher information about the parameter vector $\boldsymbol{\theta}$ in the sample $\Delta \mathbf{Y}$ is given by Fisher matrix $\mathbf{I}_{\boldsymbol{\theta}}$ :

$$
\begin{gathered}
\mathbf{I}_{\boldsymbol{\theta}}=\left(\begin{array}{ccc}
I_{\sigma_{x} \sigma_{x}} & I_{\sigma_{x} H} & I_{\sigma_{x} \sigma_{n}} \\
I_{\sigma_{x} H} & I_{H H} & I_{H \sigma_{n}} \\
I_{\sigma_{x} \sigma_{n}} & I_{H \sigma_{n}} & I_{\sigma_{n} \sigma_{n}}
\end{array}\right), \\
\text { where } I_{\theta_{i} \theta_{j}}=\frac{1}{2} \operatorname{tr}\left(\mathbf{R}_{\Delta \mathbf{Y}}^{-1} \frac{\partial \mathbf{R}_{\Delta \mathbf{Y}}}{\partial \theta_{i}} \mathbf{R}_{\Delta \mathbf{Y}}^{-1} \frac{\partial \mathbf{R}_{\Delta \mathbf{Y}}}{\partial \theta_{j}}\right) .
\end{gathered}
$$

The Cramer-Rao lower bound (CRLB) on noise STD estimate $\sigma_{\sigma_{n}}^{2}$ gives the smallest variance reachable by an unbiased estimator. In case of perfectly known Hurst exponent, $\sigma_{\sigma_{n}}^{2}$ is obtained as the element $(2,2)$ of the inverse of the matrix $\left(\begin{array}{cc}I_{\sigma_{x} \sigma_{x}} & I_{\sigma_{x} \sigma_{n}} \\ I_{\sigma_{x} \sigma_{n}} & I_{\sigma_{n} \sigma_{n}}\end{array}\right)$ :

$$
\sigma_{\sigma_{n}}^{2}=\frac{I_{\sigma_{x} \sigma_{x}}}{I_{\sigma_{x} \sigma_{x}} I_{\sigma_{n} \sigma_{n}}-2 I_{\sigma_{x} \sigma_{n}}^{2}} .
$$

When the texture Hurst exponent is known with an error $\Delta H$, noise STD estimate will be biased by

$$
\Delta \sigma=\Delta H \cdot\left(\frac{I_{\sigma_{n} H} I_{\sigma_{x} \sigma_{x}}-I_{\sigma_{n} \sigma_{x}} I_{\sigma_{x} H}}{I_{\sigma_{x} \sigma_{x}} I_{\sigma_{n} \sigma_{n}}-2 I_{\sigma_{x} \sigma_{n}}^{2}}\right) .
$$

In our case, the Hurst exponent value for a given SW is predicted from the neighboring SWs, and we assume $\Delta H$ to be zero mean random variable with variance $\sigma_{H}^{2}$. In this case, by combining (5) and variance of (6), we obtain CRLB $\sigma_{\sigma_{n}}^{2}$ through the elements of Fisher information matrix $\mathbf{I}_{\boldsymbol{\theta}}$ as

$$
\sigma_{\sigma_{n}}^{2}=\frac{I_{\sigma_{x} \sigma_{x}}}{I_{\sigma_{x} \sigma_{x}} I_{\sigma_{n} \sigma_{n}}-2 I_{\sigma_{x} \sigma_{n}}^{2}}+\sigma_{H}^{2} \cdot\left(\frac{I_{\sigma_{n} H} I_{\sigma_{x} \sigma_{x}}-I_{\sigma_{n} \sigma_{x}} I_{\sigma_{x} H}}{I_{\sigma_{x} \sigma_{x}} I_{\sigma_{n} \sigma_{n}}-2 I_{\sigma_{x} \sigma_{n}}^{2}}\right)^{2} .
$$

We propose to use relative CRLB $\sigma_{\sigma_{n}}^{2}$.rel $=\sigma_{\sigma_{n}}^{2} / \sigma_{n}^{2}$ to discriminate between NI and TI SWs as

$$
\mathrm{SW}_{\text {type }}\left(t_{0}, s_{0}\right)= \begin{cases}\text { “NI”, } & \sigma_{\sigma_{n} \cdot \text { rel } \cdot\left(t_{0}, s_{0}\right)}<\sigma_{\sigma_{n}} \cdot \text { rel } \cdot \max \\ \text { “TI”, } & \sigma_{\sigma_{n} \cdot \text { rel } \cdot\left(t_{0}, s_{0}\right)} \geq \sigma_{\sigma_{n}} \cdot \text { rel } \cdot \max .\end{cases}
$$

To set $\sigma_{\sigma_{n} \cdot \text { rel } \cdot \max }$, we require a noise STD estimation error from a single NI SW to be less than its true value: $3 \sigma_{\sigma_{n} \cdot \text { rel } \cdot \max } \sigma_{n 0}<\sigma_{n 0}$ or $\sigma_{\sigma_{n} \cdot \text { rel } \cdot \max }<1 / 3$ (otherwise, noise 


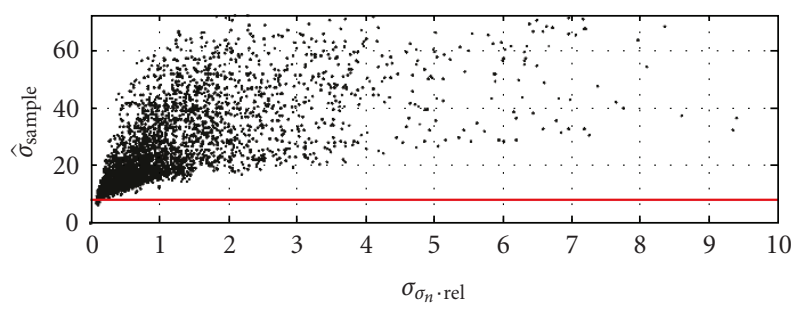

(a)

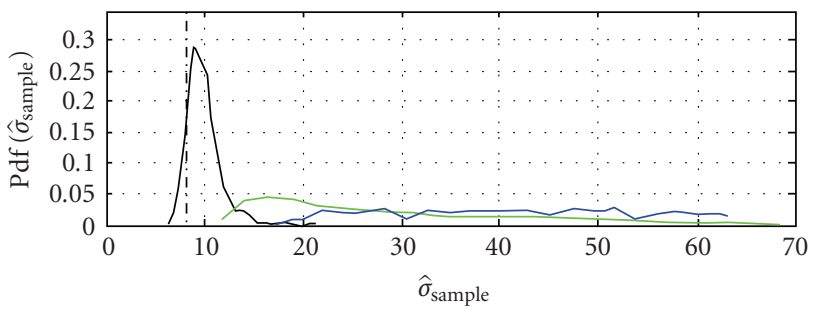

(b)

FIgURE 2: Dependence of noise STD estimates on relative CRLB $\sigma_{\sigma_{n}}^{2}$.rel for the image 14 from the TID2008 database. (a) Sample STD, $\hat{\sigma}_{\text {sample }}$, within a $7 \times 7 \mathrm{SW}$ versus $\sigma_{\sigma_{n} \text {.rel }}$; (b) distribution of $\hat{\sigma}_{\text {sample }}$ for three cases: $\sigma_{\sigma_{n}} \cdot$ rel $\leq \sigma_{\sigma_{n}}$.rel $\cdot \max \left(\right.$ black curve), $\sigma_{\sigma_{n} \cdot \text { rel }}>2 \cdot \sigma_{\sigma_{n}}$.rel $\cdot \max$ (green curve) and $\sigma_{\sigma_{n}} \cdot$ rel $>4 \cdot \sigma_{\sigma_{n}} \cdot$ rel $\cdot \max$ (blue curve).

STD estimates are expected to follow undesirable nonGaussian distribution). In our experiments, we set $\sigma_{\sigma_{n} \cdot \text { rel } \cdot \max }=0.25$.

The utility of relative CRLB $\sigma_{\sigma_{n} \text {.rel }}^{2}$ for noise STD estimation problem is illustrated in Figure 2. Figure 2(a) shows sample $\mathrm{STD}, \hat{\sigma}_{\text {sample, }}$, within a $7 \times 7 \mathrm{SW}$ versus $\sigma_{\sigma_{n}}$.rel for all nonoverlapping SWs of the test image 14 from TID2008 database (Figure 4(b); for database description see the beginning of Section 3). True noise STD used in our experiment, $\sigma_{n 0}=8.06$, is shown as horizontal red line. It can be observed that sample STD is close to the true value for small $\sigma_{\sigma_{n}}$.rel increasing fast with $\sigma_{\sigma_{n} \cdot \text { rel. }}$. Analysis of distributions of the sample STD for NI SWs $\left(\sigma_{\sigma_{n}}\right.$-rel $\leq$ $\sigma_{\sigma_{n}}$-rel - max , black plot in Figure 2(b)) and TI SWs (green and blue plots in Figure 2(b) corresponds to $\sigma_{\sigma_{n}}$.rel $>2 \cdot \sigma_{\sigma_{n}}$.rel $\cdot \max$ and $\sigma_{\sigma_{n}}$.rel $>4 \cdot \sigma_{\sigma_{n}}$.rel $\cdot$ max , resp.) shows that TI SWs should not be used for noise STD estimation. On the contrary, the distribution for NI SWs is close to the desirable one: it is centered close to the true value (marked by dashed vertical line) and with significantly shorter right-hand tail.

2.4. ML Estimators for Texture Parameters and Noise STD. To implement the detector (8), the parameter vector $\boldsymbol{\theta}$ is to be estimated for the whole image (for both NI and TI SWs). The goal of the first stage of our algorithm is to estimate $\boldsymbol{\theta}$ for SWs that belong to TI map. As TI SWs do not provide information on noise STD, we fix noise STD equal to either an initial guess $\hat{\sigma}_{n}=\hat{\sigma}_{n \cdot i=0}$ or previously estimated value $\hat{\sigma}_{n}=\hat{\sigma}_{n \cdot i-1}$. Note that in the additive noise case, $\hat{\sigma}_{n}$ is the same for all SWs. Hereafter, index $i$ denotes a current iteration of the algorithm. Initial guess for the estimation $\hat{\sigma}_{n \cdot i=0}$ can be obtained as the minimum of sample STD estimates over all image nonoverlapping SWs.
The ML estimator of fBm-model parameters, $H$ and $\sigma_{x}$, in a single TI SW ( $\mathrm{SW}_{\mathrm{type} \cdot i-1}\left(t_{0}, s_{0}\right)=$ "TI") is given as

$$
\left[\hat{\sigma}_{x \cdot\left(t_{0}, s_{0}\right) \cdot i}, \hat{H}_{\left(t_{0}, s_{0}\right) \cdot i}, \sigma_{n}=\hat{\sigma}_{n}\right]=\underset{\sigma_{x} \geq 0,0 \leq H \leq 1}{\arg \min }[\ln L(\Delta \mathbf{Y} ; \boldsymbol{\theta})] .
$$

Here $\mathrm{SW}_{\text {type } \cdot i-1}\left(t_{0}, s_{0}\right)$ is an estimate of informative map obtained at the previous iteration $i-1$. Initially, when $i=0$, the entire image is considered to be TI. Thus, for each TI SW we estimate the parameter vector as $\widehat{\boldsymbol{\theta}}_{\mathrm{TI}}=$ $\left(\hat{\sigma}_{x \cdot\left(t_{0}, s_{0}\right) \cdot i}, \hat{H}_{\left(t_{0}, s_{0}\right) \cdot i}, \hat{\sigma}_{n}\right)$.

Next, the goal of the second stage is to estimate $\boldsymbol{\theta}$ for all NI SWs ( $\operatorname{SW}_{\text {type } i-1}\left(t_{0}, s_{0}\right)=$ "NI"). In this case, we can use the estimate $\widehat{\sigma}_{n}=\widehat{\sigma}_{n \cdot i-1}$ for noise STD as well. Following the discussion above, the Hurst exponent, $\hat{H}_{\mathrm{pr} \cdot\left(t_{0}, s_{0}\right) \cdot i}$, in the NI SWs centered at $\left(t_{0}, s_{0}\right)$ is predicted from all current TI SWs in the neighborhood of pixel $\left(t_{0}, s_{0}\right)$ by simple averaging:

$$
\hat{H}_{\mathrm{pr} \cdot\left(t_{0}, s_{0}\right) \cdot i}=\frac{1}{|\Omega|} \sum_{(t, s) \in \Omega} \hat{H}_{\left(t_{0}, s_{0}\right) \cdot i},
$$

where $\Omega=\left\{(t, s), t \neq t_{0}, s \neq s_{0},\left|t-t_{0}\right|<N_{a},\left|s-s_{0}\right|<N_{a}\right.$, $\mathrm{SW}_{\text {type } \cdot i-1}(t, s)=$ "TI" $\}, N_{a}$ limits the averaging support. We select $N_{a}=2 \cdot N$ meaning that $5 \times 5$ nonoverlapping SWs are used. In case $|\Omega|=0$, we fix $\hat{H}_{\mathrm{pr} \cdot\left(t_{0}, s_{0}\right) \cdot i}=\hat{H}_{\mathrm{TI} \text {-mean }}$, where $\hat{H}_{\mathrm{TI}}$-mean is the Hurst exponent estimates average over all current TI SWs.

It is assumed that the value $H$ describing texture roughness is slowly varying with spatial coordinates (e.g., the same $H$ value can be used to describe a large uniform textural area) and, thus, it can be predicted by (10). On the contrary, $\sigma_{x}$ describing texture amplitude can vary significantly from SW to SW and should be estimated directly from the data as

$$
\begin{gathered}
\left(\hat{\sigma}_{x \cdot\left(t_{0}, s_{0}\right) \cdot i}, H=\hat{H}_{\mathrm{pr} \cdot\left(t_{0}, s_{0}\right) \cdot i}, \sigma_{n}=\hat{\sigma}_{n \cdot i-1}\right) \\
=\underset{\sigma_{x} \geq 0}{\arg \min }[\ln L(\Delta \mathbf{Y} ; \boldsymbol{\theta})] .
\end{gathered}
$$

As a result, for each NI SW we estimate the parameter vector as $\hat{\boldsymbol{\theta}}_{\mathrm{NI}}=\left(\hat{\sigma}_{x \cdot\left(t_{0}, s_{0}\right) \cdot i}, \hat{H}_{\mathrm{pr} \cdot\left(t_{0}, s_{0}\right) \cdot i}, \hat{\sigma}_{n}\right)$.

At this stage, we have $\boldsymbol{\theta}$ estimated for the whole image. To estimate the CRLB $\hat{\sigma}_{\sigma_{n} \cdot\left(t_{0}, s_{0}\right) \cdot i}^{2}$ by $(7)$, the value of $\sigma_{H}^{2}$ still remains unknown. This value can be estimated from TI map by taking variance of difference between the Hurst exponent estimates obtained by (9) and (10):

$$
\sigma_{H \cdot i}^{2}=D\left(\hat{H}_{\mathrm{pr} \cdot\left(t_{0}, s_{0}\right) \cdot i}-\hat{H}_{\left(t_{0}, s_{0}\right) \cdot i}\right),
$$

where $D(\cdot)$ is the variance operator and variance is calculated over all current TI SWs $\left(\mathrm{SW}_{\text {type } \cdot i-1}\left(t_{0}, s_{0}\right)=\right.$ "TI").

Having vector $\boldsymbol{\theta}$ estimated for all NI and TI SWs, we obtain CRLB $\hat{\sigma}_{\sigma_{n} \cdot\left(t_{0}, s_{0}\right) \cdot i}^{2}$ in these SWs by substituting $\boldsymbol{\theta}=\widehat{\boldsymbol{\theta}}_{\mathrm{TI}}$ or $\boldsymbol{\theta}=\widehat{\boldsymbol{\theta}}_{\mathrm{NI}}$ and $\sigma_{H}^{2}=\sigma_{H \cdot i}^{2}$ into (4) and (7). Finally, we can update discriminative map $\mathrm{SW}_{\text {type } \cdot i-1}\left(t_{0}, s_{0}\right)$ obtained at the previous stage to current $\mathrm{SW}_{\text {type } \cdot i}\left(t_{0}, s_{0}\right)$ map by $(8)$.

The goal of the next stage is to estimate image noise STD from NI SWs. Texture parameters are fixed and equal 
to the previously estimated vector $\hat{\boldsymbol{\theta}}_{\mathrm{NI}}$. For the updated $\mathrm{SW}_{\text {type } \cdot i}\left(t_{0}, s_{0}\right)$ map, we define the ML estimator of noise STD in a single NI SW (SW $\mathrm{SWpe}_{\text {ty } i}\left(t_{0}, s_{0}\right)=$ "NI") as

$$
\left(\hat{\sigma}_{x \cdot\left(t_{0}, s_{0}\right) \cdot i}, H=\hat{H}_{\mathrm{pr} \cdot\left(t_{0}, s_{0}\right) \cdot i}, \widehat{\sigma}_{n \cdot\left(t_{0}, s_{0}\right) \cdot i}\right)=\underset{\sigma_{x} \geq 0, \sigma_{n} \geq 0}{\arg \min }[\ln L(\Delta \mathbf{Y} ; \boldsymbol{\theta})] .
$$

To solve constrained optimization problems (9), (11), and (13), we have used the Han-Powell optimization method described in [32]. This method belongs to the quasiNewton group and, therefore, it provides high (superlinear) convergence speed which is important for the considered application. In addition, this method includes quadratic programming step for which efficient standard procedures are available.

Finally, we update the current noise STD estimate for the whole image by calculating a weighted average over all current NI SWs:

$$
\begin{gathered}
\hat{\sigma}_{n \cdot i}=\frac{\left(\sum \mathrm{SW}_{\text {type } \cdot i}\left(t_{0}, s_{0}\right)=" \mathrm{NI} "\left(\hat{\sigma}_{n \cdot\left(t_{0}, s_{0}\right) \cdot i} / \hat{\sigma}_{\sigma_{n} \cdot\left(t_{0}, s_{0}\right) \cdot i}^{2}\right)\right)}{\hat{\sigma}_{\sigma_{n} \cdot \mathrm{NI}}^{2}}, \\
\hat{\sigma}_{\sigma_{n} \cdot \mathrm{NI}}^{2}=\left(\sum_{\mathrm{SW}_{\text {type } \cdot i}\left(t_{0}, s_{0}\right)=\text { "NI" }} \hat{\sigma}_{\sigma_{n} \cdot\left(t_{0}, s_{0}\right) \cdot i}^{-2}\right)^{-1},
\end{gathered}
$$

where $\hat{\sigma}_{\sigma_{n} \cdot \mathrm{NI}}^{2}$ is estimated CRLB on additive noise STD estimate $\hat{\sigma}_{n \cdot i}$ from the whole NI map.

For comparison purpose, in addition to the $\mathrm{fBm}$-based estimator (14), let us consider also DCT-based estimator at STD estimation stage of our algorithm. STD estimators based on DCT have demonstrated themselves to be quite accurate for high complexity images [14, 33], that is why we expect this approach to perform well within our framework. The additionally proposed estimator performs as follows. For each NI SW, we apply $7 \times 7$ DCT and consider only the six highest frequency coefficients with indices $(7,7),(6,7)$, $(7,6),(6,6),(5,7)$, and $(7,5)$. These DCT coefficients are almost insensitive to image content because of two reasons. First, they are calculated for NI SWs. Second, high frequency components of orthogonal transforms are known to be less influenced by image content than low frequency ones [15]. These DCT coefficients are collected from all NI scanning windows $\left(\mathrm{SW}_{\text {type } \cdot i}\left(t_{0}, s_{0}\right)=\right.$ "NI") and the final noise STD estimate, $\hat{\sigma}_{n \cdot i}$, is obtained as sample STD of the formed array of DCT coefficients.

The use of six coefficients allows estimating noise STD in each SW with fixed relative STD $1 / \sqrt{2 \cdot 6} \approx 0.29$. This is in agreement with the earlier selected threshold $\sigma_{\sigma_{n}} \cdot$ rel $\cdot \max =$ 0.25 .

Before proceeding further, we would like to emphasize the meaning and practical importance of $\hat{\sigma}_{\sigma_{n} \cdot \text { NI }}^{2}$ value provided within our scheme. On one hand, it establishes one possible theoretical lower bound on noise STD estimate variance from a given image. On the other hand, it can be estimated directly from a noisy image making it practically interesting since no reference image is needed for deriving $\hat{\sigma}_{\sigma_{n}}^{2} \cdot \mathrm{NI}$. In the experimental part of the paper, we will provide

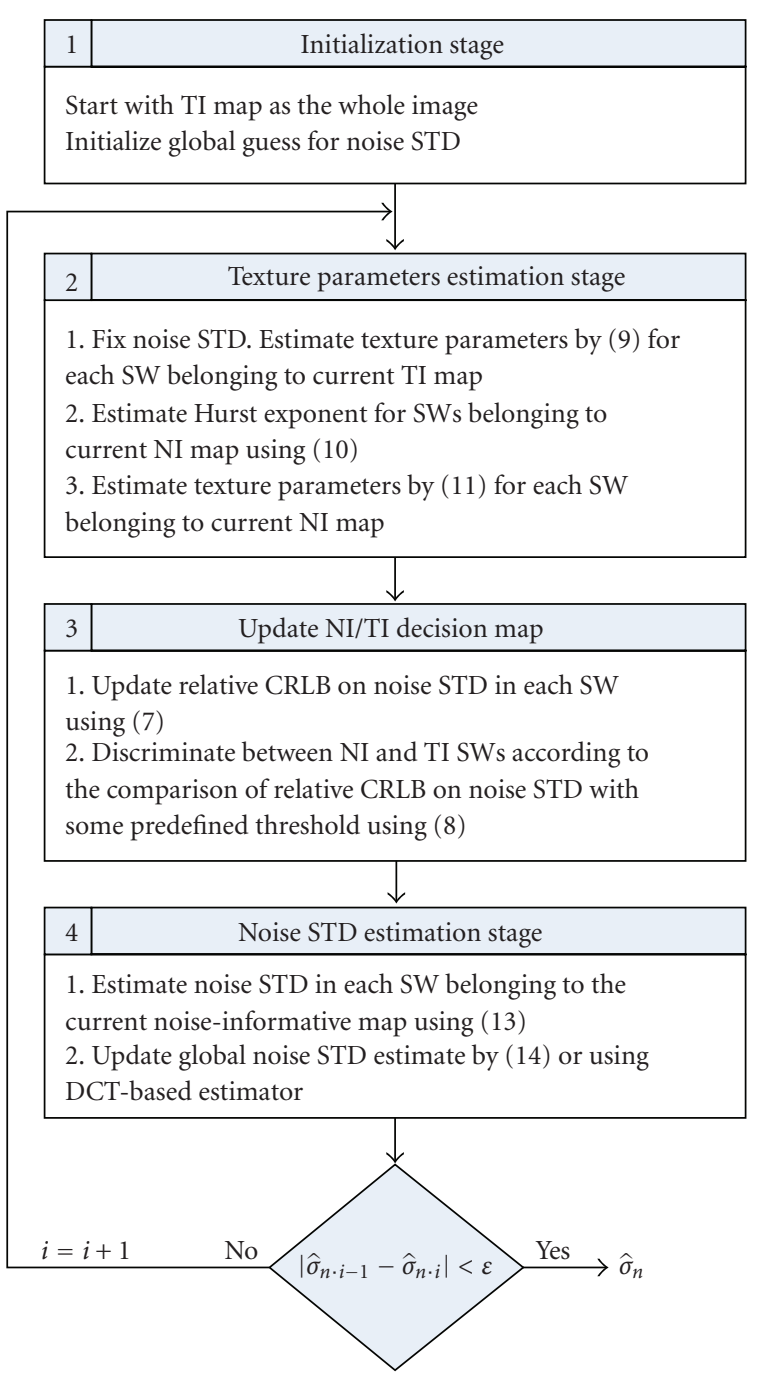

FIGURE 3: Generalized scheme of the proposed iterative noise STD estimation algorithm.

some results showing how close to this bound our and two state-of-the-art noise STD estimators (BM3D and SBIQ, see details in Section 3) are.

2.5. Estimator Structure and Convergence. The generalized scheme of the proposed iterative noise STD estimation algorithm is given in Figure 3.

To understand the algorithm convergence, let us consider an extreme case for which noise intensity is negligible with respect to texture intensity in TI areas (local SNR approaches infinity where local SNR is defined as the $\sigma_{x}^{2} / \sigma_{n}^{2}$ ratio) and texture intensity is negligible with respect to noise intensity in NI areas (local SNR approaches zero). Then, an error in initial noise STD estimation slightly influences the Hurst exponent estimates obtained by (9) and (10). In turn, small error in $H$ estimates have little effect on noise STD estimates derived according to (13) and (14) or by using DCT. Therefore, after one algorithm iteration, initial noise STD error reduces and rapidly converges with iterations. In the more realistic case, for which local SNRs in TI and 
NI areas take high and low values with respect to unity, convergence is also reached but with a smaller rate.

\section{Accuracy Analysis of Noise STD Estimation Using TID2008 Database}

For analysis purpose, the database TID2008 [34] was chosen (available at http://ponomarenko.info/tid2008.htm). This choice is mainly explained by the following reasons. First, TID2008 comprises 25 color images with low level of selfnoise. 24 images are taken from Kodak image database http://r0k.us/graphics/kodak/ and one is artificially synthesized (the 25th). All images are of equal size of $512 \times$ 384 pixels. The database includes images with different content. Some of them have quite large homogeneous areas, the others are quite textural. This allows testing the proposed estimator for images with different content. Second, the database includes images corrupted by additive white Gaussian noise with variance 65, 130, 260, and 520 . From practical point of view, the first two cases are of interest. The noisy images with smaller noise variance values can be generated based on practically noise-free reference images provided in the database. Third, the TID2008 database has been used to evaluate performance of several state-of-the-art noise STD estimators $[10,14,35]$. Thus, the testing results reported previously can be used for comparisons.

In this section, we analyze performance of two versions of the proposed noise STD estimator (later referred to as $\mathrm{NI}+\mathrm{fBm}$ or NI+DCT depending on which algorithm is used for noise STD estimation from NI map: $\mathrm{fBm}$ - or DCT based). Their respective performance is then compared to two state-of-the-art estimators: the BM3D estimator based on nonlocal 3D DCT transform [14] and the segmentationbased interquantile estimator (below it is referred to as SBIQ [35]). Here we would like to thank A. Foi for passing us the results for the BM3D estimator for noise variance equal to 25 and 130 .

Color images in TID2008 are represented by 24 bit data arrays, so for each color component the image values are bounded above and below by $I_{\max }=255$ and $I_{\min }=0$, respectively. As a result, noisy images in significantly dark or bright areas become clipped. As clipping effect deviates noise distribution from Gaussian and makes its variance smaller, this leads to negatively biased noise STD estimates [36]. To alleviate this effect, we have detected and removed from further consideration SWs affected by clipping effects. This has been carried out using the following rule: a SW is rejected if more than $10 \%$ of its pixels have intensity equal to $I_{\min }$ or $I_{\max }$.

Let us start by presenting examples of NI and TI maps obtained at convergence (4 iterations were needed to obtain these maps). Figures 4(a) and 4(b) give examples for noisy test images 13 and $14\left(\sigma_{n 0}^{2}=65\right)$, respectively. Color and gray tones are associated to NI and TI SWs, respectively. One can see that the NI maps are mainly comprised of HAs (sky in Figure 4(a) and boat surface in Figure 4(b)) and areas with nonintensive texture (nonintensive forest pattern in Figure 4(a) and water surface in Figures 4(a) and 4(b)). On the contrary, TI maps include textural areas (intensive forest pattern in Figure 4(a) and intensive water pattern in Figures $4(\mathrm{a})$ and $4(\mathrm{~b}))$ and edges.

The Hurst exponent estimates, $\hat{H}_{r \cdot\left(t_{0}, s_{0}\right)}$, obtained for the reference image 13 (considered as noise-free) are displayed as a map in Figure 4(c). Figure 4(d) displays the corresponding $\hat{H}_{\mathrm{pr} \cdot\left(t_{0}, s_{0}\right)}$ map with areas affected by the clipping effect shown as black. It can be well observed that $\hat{H}_{\mathrm{pr} \cdot\left(t_{0}, s_{0}\right)}$ map is a smoothed version of the $\hat{H}_{r \cdot\left(t_{0}, s_{0}\right)}$ map. Similar observations hold for other images from TID2008 database. These results demonstrate possibility of using (10) for $H$ prediction in NI areas.

The properties of NI map are illustrated in Figure 5 for the image 14 and $\sigma_{n 0}^{2}=65$. First, Figure 5(a) compares the empirical probability density functions (pdf) of two noise local STD estimates obtained for $7 \times 7$ NI SWs. The first pdf is for $\mathrm{fBm}$-based estimates $\hat{\sigma}_{x \cdot\left(t_{0}, s_{0}\right)}$ given by (13) (shown in black color). The second pdf relates to sample STD estimates for the six highest DCT frequency coefficients, $\hat{\sigma}_{\mathrm{DCT}} \cdot\left(t_{0}, s_{0}\right)$ (shown in green color). The third pdf corresponds to the standard sample STD (shown in blue color).

As it is seen, the standard sample STD pdf is significantly shifted with respect to the true value of noise STD. This is due to influence of low intensity texture (there are practically no really homogeneous areas in real-life images). Both fBmand DCT-based estimates are almost unbiased. The only difference is that the $\mathrm{fBm}$-based estimates have notably smaller variance compared to the DCT-based estimates. To highlight this difference, Figure 5(b) shows the corresponding empirical pdfs of noise STD estimates normalized by

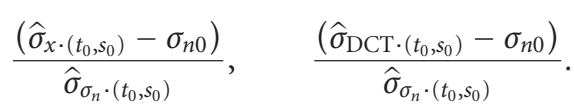

The normalized estimates can be considered as Gaussianlike distributed random variables with variance close to unity for the $\mathrm{fBm}$-based estimates (theoretical pdf $N(0,1)$ is shown for comparison as red curve). Estimation variance for DCTbased method is about 2.5 times larger. This difference in estimation accuracy is explained by the fact that the DCTbased estimator uses only six coefficients in each NI SW. Thus, in homogeneous NI SWs, where up to $7 \cdot 7=49$ pixels can be used for noise STD estimation in $7 \times 7 \mathrm{SW}$, accuracy of the DCT-based estimator is lower than CRLB $\hat{\sigma}_{\sigma_{n}} \cdot\left(t_{0}, s_{0}\right)$. On the contrary, our fBm-based estimator allows estimating noise STD with accuracy close to CRLB $\hat{\sigma}_{\sigma_{n} \cdot\left(t_{0}, s_{0}\right)}$ in each NI SW.

As the estimated CRLB value, $\hat{\sigma}_{\sigma_{n} \cdot\left(t_{0}, s_{0}\right)}$, is close to the actual STD of $\hat{\sigma}_{x \cdot\left(t_{0}, s_{0}\right)}$ estimates in each NI SWs, we can expect CRLB $\hat{\sigma}_{\sigma_{n}}^{2}$-NI $(14)$ to be a valid estimate of the potential variance of the global noise STD estimate that can be obtained from NI maps.

One can expect that the accuracy for all methods depends upon image complexity and noise variance. Image complexity can be, in particular, characterized by the number of detected NI SWs $N_{\mathrm{NI}}$ or, more generally, by the ratio $N_{\mathrm{NI}}$. rel $=$ $N_{\mathrm{NI}} / N_{\mathrm{SW}}$, where $N_{\mathrm{SW}}$ is the total number of nonoverlapping SWs. Thus, let us consider $N_{N I}$.rel for different images in 


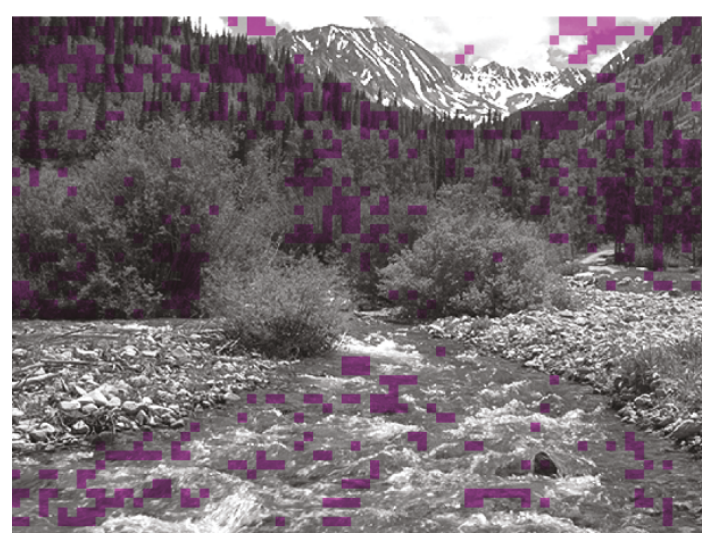

(a)

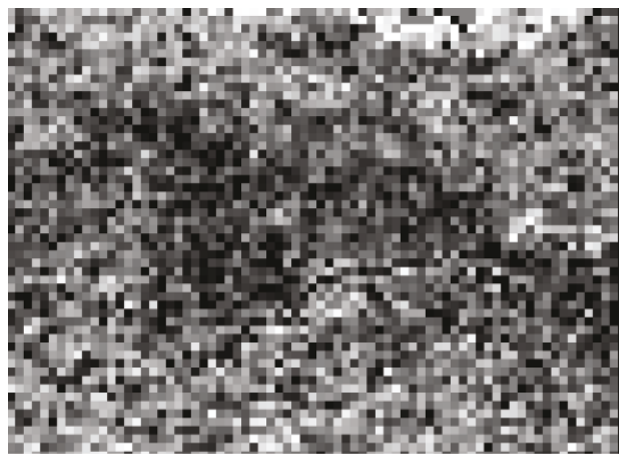

(c)

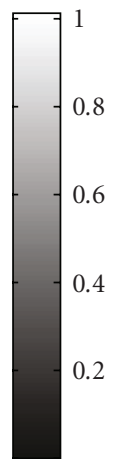

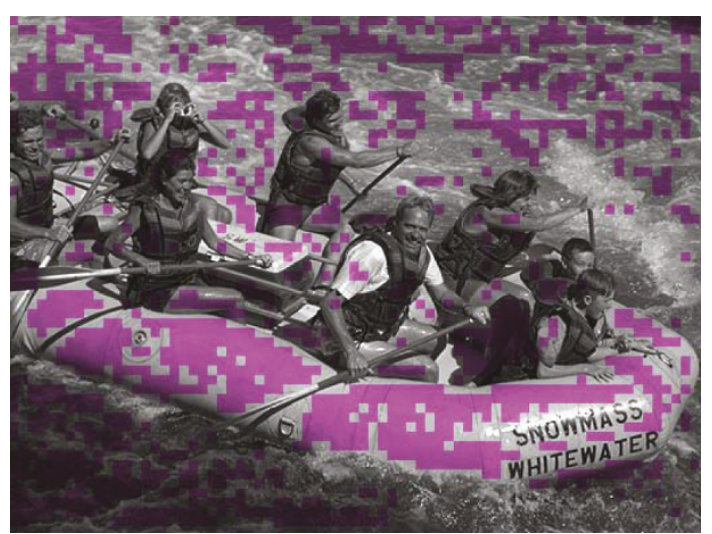

(b)

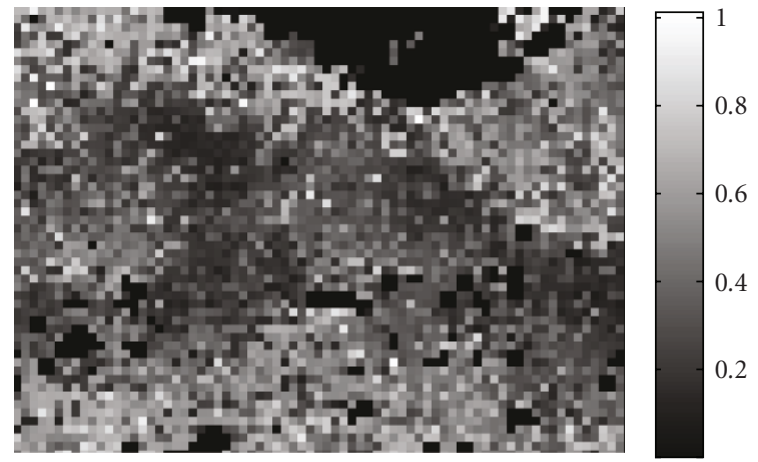

(d)

FIGURE 4: NI maps for the test images 13 and 14 (NI SWs are marked by magenta, TI are in gray tones, $\sigma_{n 0}^{2}=65$ ) (a) and (b); $\hat{H}_{r}$ map for the test image 13 , black and white colors corresponds to $H=0$ and $H=1$, respectively, (c); $\hat{H}_{\text {pr }}$ map for noisy image $13, \sigma_{n 0}^{2}=65$ (d).

TID2008 keeping in mind that the larger $N_{\mathrm{NI}}$.rel should produce better accuracy of noise variance estimation.

Values $N_{\mathrm{NI}}$.rel for all images of TID2008 database and for $\sigma_{n 0}^{2}=25,65$ and 130 are shown in Figure $6 . N_{\text {NI. rel }}$ varies from $80 \%$ for images comprised of large homogeneous areas like $(3,4$, or 23$)$ to $1 \%$ for the highly textural image 13 . This explains why it is difficult to provide high accuracy of blind estimation of noise STD for textural images.

Note that NI map also depends on noise variance. In general, $N_{\mathrm{NI}}$ increases with $\sigma_{n 0}^{2}$ due to reduced influence of image texture. For example, if $\sigma_{n 0}^{2}$ increases from 25 to 130 , $N_{\mathrm{NI}}$ on the average increases by 2.2 times (in particular, by 11 times for image 13 and by 1.02 times for the synthetic image 25). As a result, we expect noise variance estimators to be more efficient for larger $\sigma_{n 0}^{2}$. This fact is well known in practice for different estimators [10].

Within the proposed approach, the CRLB $\hat{\sigma}_{\sigma_{n} \cdot \mathrm{NI}}^{2}$ can characterize this tendency quantitatively. Let us consider the relative CRLB $\sigma_{\sigma_{n} \text {-NI.rel }}^{2}=\sigma_{\sigma_{n} \text {-NI }}^{2} / \sigma_{n 0}^{2}$. Under assumption of Gaussian distribution of noise STD estimates and their unbiasedness, relative CRLB determines potential 99.7\% interval for $\hat{\sigma}_{n}$ as $\hat{\sigma}_{n} \in \sigma_{n 0} \cdot\left[1-3 \cdot \sigma_{\sigma_{n} \cdot \mathrm{NI} \cdot \text { rel }}, 1+3 \cdot \sigma_{\sigma_{n} \cdot \mathrm{NI} \cdot \text { rel }}\right]$. The values of $\sigma_{\sigma_{n}}$-NI.rel obtained for images in TID2008 database are given in Figure 7. It can be observed from this figure that $\sigma_{\sigma_{n}}$.NI.rel steadily decreases if $\sigma_{n 0}^{2}$ becomes larger. For example, with $\sigma_{n 0}^{2}$ increasing from 25 to $130, \sigma_{\sigma_{n}}$-NI. rel, on the average, reduces by 1.6 times. Averaging above is done for all images of the considered database. Average values of $\sigma_{\sigma_{n}}$.NI.rel are equal to $0.27 \%$ for $\sigma_{n}^{2}=130$ and to $0.45 \%$ for $\sigma_{n}^{2}=25$. This means that for $\sigma_{n 0}^{2}=130$, noise STD estimates in the ideal case should belong to a very narrow range $11.4 \cdot[1-3 \cdot 0.0027,1+3 \cdot 0.0027] \approx[11.3,11.5]$. Similarly, for $\sigma_{n 0}^{2}=25$ this interval becomes $5 \cdot[1-3$. $0.0045,1+3 \cdot 0.0045] \approx[4.93,5.07]$.

Let us now test the performance of the $\mathrm{NI}+\mathrm{fBm}$, NI+DCT, BM3D, and SBIQ noise STD estimators for all images of the TID2008 database. Initially, our estimator has been applied to all 25 reference images of the TID2008 database in order to estimate noise that originally affects the database images considered almost noise-free. The results of noise STD estimation by NI+DCT for reference images, $\hat{\sigma}_{r}$, are consistent for all considered images, varying from 0.3 to 2 .

In Figure 8, convergence of NI+DCT estimator for the red channel of image 14 is shown. The true noise STD is $\sigma_{n 0}=8.06\left(\sigma_{n 0}^{2}=65\right)$ and it is marked by dashed thin horizontal line. The black curve corresponds to the situation when initial guess $\hat{\sigma}_{n \cdot i=0}$ was selected as described above in Section 2: the minimal sample STD estimate over all image nonoverlapping SWs has been used as $\hat{\sigma}_{n \cdot i=0}$. In this case, five iterations are needed for the algorithm to converge from initial guess $\left(\hat{\sigma}_{n \cdot i=0} \approx 7.0\right)$ to the final STD value 8.15. 


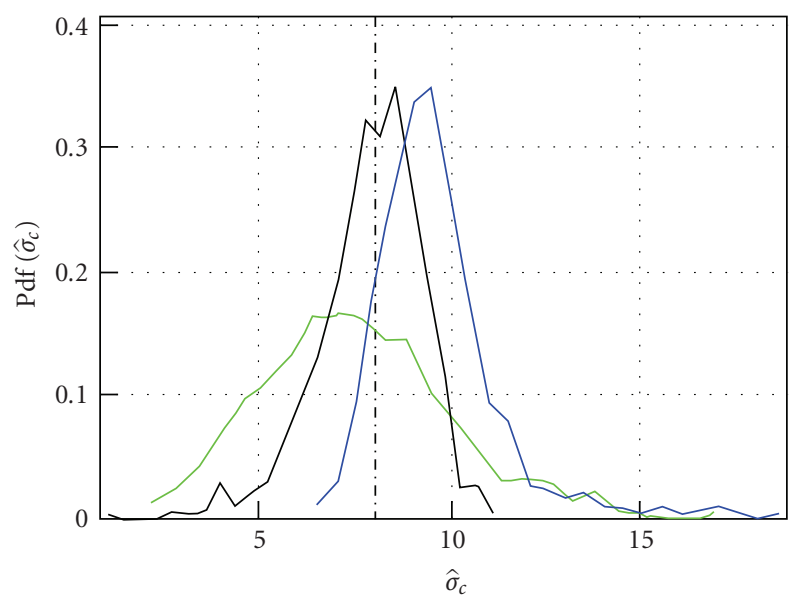

(a)

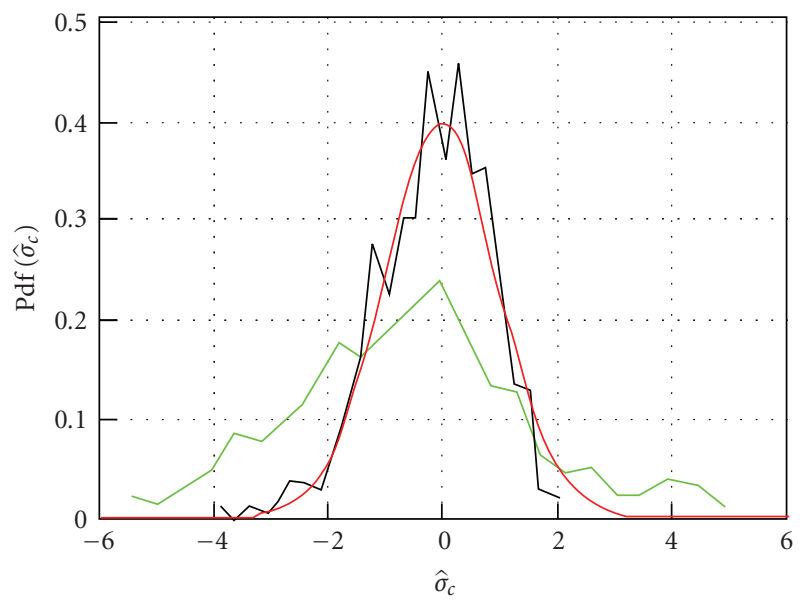

(b)

FIGURE 5: Distributions (empirical pdfs) of the noise STD estimates for $7 \times 7$ NI SWs: (a) pdf of $\hat{\sigma}_{x \cdot\left(t_{0}, s_{0}\right)}$ estimates (black curve); pdf of sample STD for the DCT based method (green curve); pdf of standard sample STD (green curve). The true value $\sigma_{n 0}$ is marked as dashed vertical line; (b) pdf of the normalized noise STD estimates for $\mathrm{fBm}$ and DCT-based estimators. True noise STD is marked by dashed vertical line.

Experiments show that our algorithm converges in 3 to 6 iterations in most cases. Texture parameters estimation is the most computationally intensive part as the corresponding parameters need to be estimated for the whole image: the total time cost for 384 by 512 image on Intel Core (TM) 2 Duo $(1.66 \mathrm{GHz}) \mathrm{CPU}$ varies from 3 to 10 minutes depending on image complexity.

To demonstrate the robustness of the estimator with respect to possible large initial error of noise STD estimation, the same estimator was tested for two other initial guesses: $\hat{\sigma}_{n \cdot i=0}=16.0$ and $\hat{\sigma}_{n \cdot i=0}=4.0$. In both cases, the estimator has converged to the same noise STD final estimate, but it has taken more iterations (six iterations instead of four).

Next, we have considered additive noise case with three different noise STDs: $\sigma_{n 0}^{2}=25,65$ and 130. Recall that for the noisy images for $\sigma_{n 0}^{2}=65$ and $\sigma_{n 0}^{2}=130$ are directly available from the TID2008 database. The noisy images for

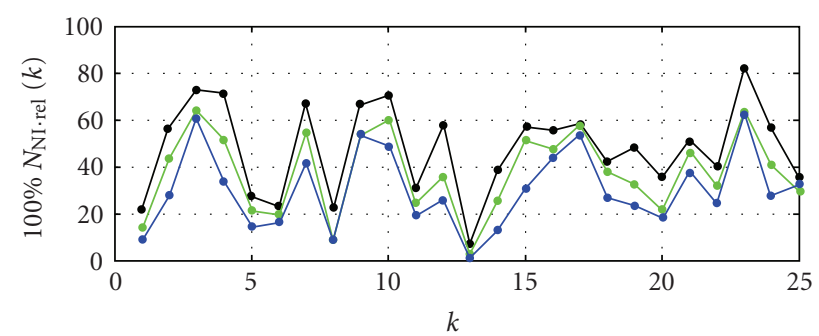

FIgURE 6: The number of NI SWs, $N_{\mathrm{NI}}$ rel versus image index $k$ for the TID2008 database for noise variance $\sigma_{n 0}^{2}=25$ (blue curve), 65 (red curve) and 130 (black curve).

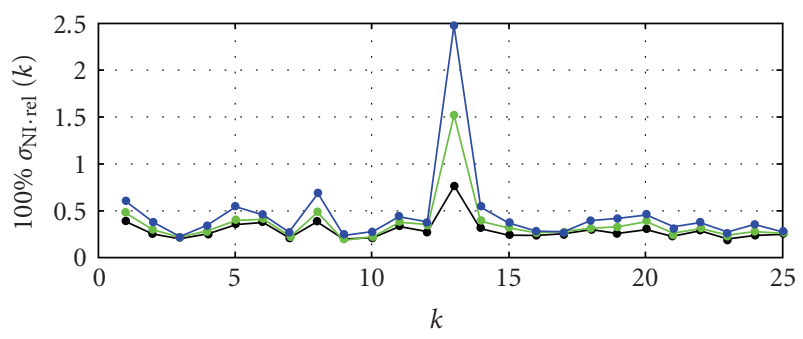

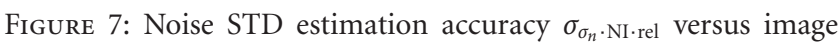
index $k$ for the TID2008 database for noise variance $\sigma_{n 0}^{2}$ equal to 25 (blue curve), 65 (red curve), and 130 (black curve).

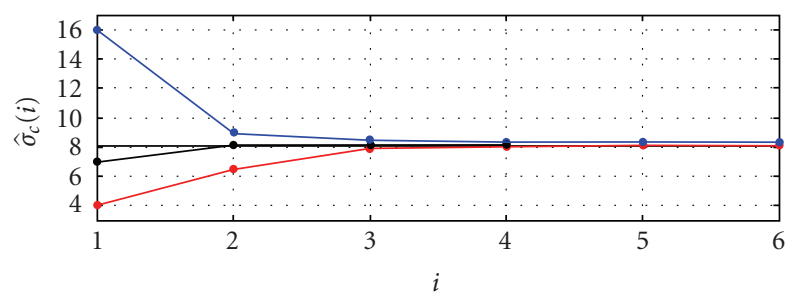

FIgure 8: Convergence of NI+DCT noise STD estimator. Initial guess for noise STD $\hat{\sigma}_{n \cdot i=0} \approx 7.0$ (black curve), $\hat{\sigma}_{n \cdot i=0}=16.0$ (blue curve) and $\hat{\sigma}_{n \cdot i=0}=4.0$ (red curve).

$\sigma_{n 0}^{2}=25$ have been generated by adding synthetic noise with the corresponding variance followed by quantization and clipping to the range from 0 to 255 . As the obtained noisy images contain both the reference images noise and the synthetic noise subsequently added, the resulting noise STD is slightly larger than $\sigma_{n 0}$. Therefore, the estimates for all considered methods have been corrected as $\hat{\sigma}_{c}=\sqrt{\hat{\sigma}^{2}-\hat{\sigma}_{r}^{2}}$.

The empirical pdfs of the obtained estimates are presented in Figure 9 for three noise variances for the proposed $\mathrm{NI}+\mathrm{fBm}$ and NI+DCT estimators (in black and red colors, resp.), the BM3D estimator (in green color) and SBIQ estimator (in blue color). The corresponding mean $\left(\operatorname{Mean}\left(\hat{\sigma}_{c}\right)\right)$ and $\operatorname{STD}\left(\operatorname{STD}\left(\hat{\sigma}_{c}\right)\right)$ of the obtained estimates over the whole TID2008 database are given in Table 1.

Analysis of data in Table 1 and Figure 9 shows that all estimators provide quite accurate estimates. The SBIQ estimator produces outlying estimates (not shown in Figure 9 but taken into account in Table 1) for the textural image 13 and images 6 and 20. The latter outliers are mainly due to clipping effect influence [35]. 
TABLE 1: Mean and STD of additive noise STD estimates $\hat{\sigma}_{c}$ for TID2008 database.

\begin{tabular}{lccc}
\hline Mean $\left(\hat{\sigma}_{c}\right) / \operatorname{STD}\left(\hat{\sigma}_{c}\right)$ & $\sigma_{n 0}^{2}=25\left(\sigma_{n 0}=5\right)$ & $\sigma_{n 0}^{2}=65\left(\sigma_{n 0}=8.06\right)$ & $\sigma_{n 0}^{2}=130\left(\sigma_{n 0}=11.40\right)$ \\
\hline NI+fBm & $5.00 / 0.120$ & $7.96 / 0.156$ & $11.35 / 0.183$ \\
NI+DCT & $5.01 / 0.060$ & $8.05 / 0.098$ & $11.41 / 0.121$ \\
BM3D & $5.06 / 0.128$ & -no data available- & $11.50 / 0.196$ \\
SBIQ & -no data available- & $8.29 / 1.372$ & $11.29 / 1.59$ \\
\hline
\end{tabular}

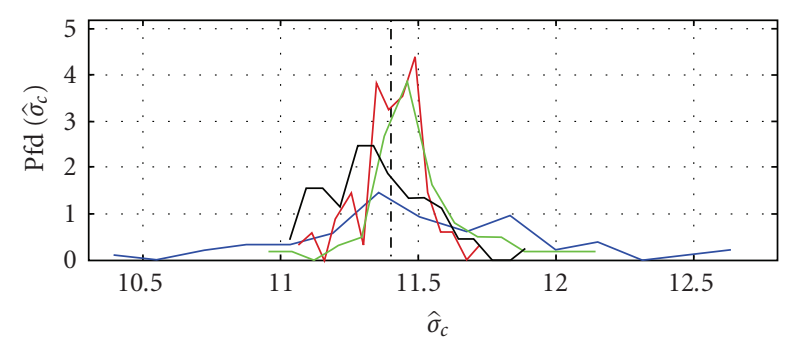

(a)

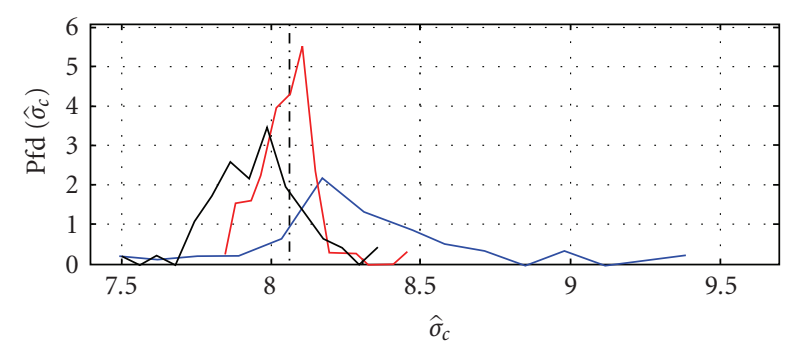

(b)

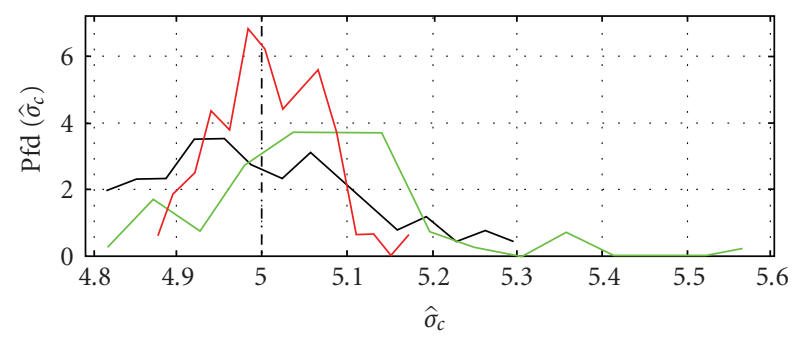

(c)

FIGURE 9: Empirical pdfs of STD estimates $\hat{\sigma}_{c}$ obtained for the whole TID2008 database by (1) NI+fBm (in black color); (2) NI+DCT (in red color); (3) BM3D (in green color); (4) SBIQ (in blue color); noise variances are equal to $\sigma_{n 0}^{2}=130(\mathrm{a}), \sigma_{n 0}^{2}=65(\mathrm{~b})$, and $\sigma_{n 0}^{2}=25$ (c). The true noise STD is marked by dashed vertical line in all plots.

According to Table 1, for the SBIQ estimator the STD $\left(\hat{\sigma}_{c}\right)$ takes the largest value $\approx 1.3-1.5$ and Mean $\left(\hat{\sigma}_{c}\right)$ is biased by about $5 \%$ (for $\sigma_{n 0}^{2}=65$ ). The BM3D and NI+fBm estimators show similar performance, reducing $\operatorname{STD}\left(\hat{\sigma}_{c}\right)$ by about $5-$ 8 times compared to SBIQ. Mean $\left(\hat{\sigma}_{c}\right)$ is biased by only $\approx 1 \%$. The NI+DCT estimator improves these results even further. $\operatorname{STD}\left(\hat{\sigma}_{c}\right)$ reduces by about $1.5-2$ times as compared to its value for $\mathrm{BM} 3 \mathrm{D}$ and $\mathrm{NI}+\mathrm{fBm}$; mean $\left(\hat{\sigma}_{c}\right)$ bias becomes negligible $(<0.4 \%)$. Note that for the NI+DCT estimator the actual ranges of $\hat{\sigma}_{c}$ variation are only approximately 2 times

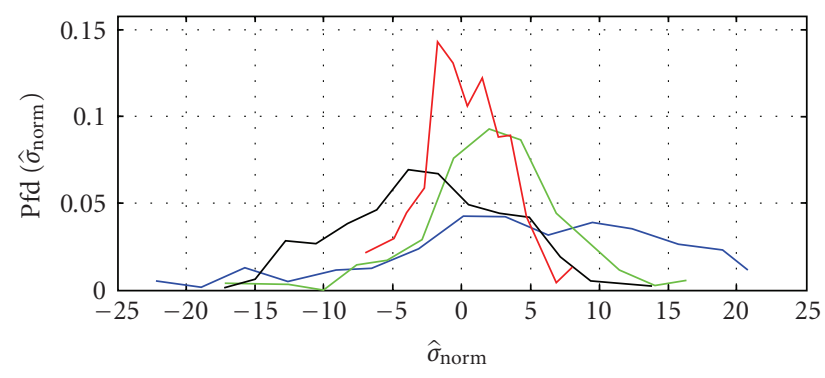

FIgURE 10: The empirical pdfs of the normalized noise STD estimates $\hat{\sigma}_{\text {norm }}$. Color settings are as in Figure 9.

wider than the ones determined by CRLB ([11.3, 11.5] for $\sigma_{n 0}^{2}=130$ and $[4.93,5.07]$ for $\left.\sigma_{n 0}^{2}=25\right)$. Thus, very accurate estimation is provided for all images of the considered database irrespectively to noise STD value.

Potentially the method $\mathrm{NI}+\mathrm{fBm}$ is expected to outperform NI+DCT estimator (see Figure 5). However, the simulation results have not shown this. The reason seems to be the following. Within the NI+fBm approach, both noise STD and Hurst exponent are estimated jointly with mutual influence on each other. Then, the errors in Hurst exponent prediction lead to additional errors of STD estimation, which, consequently, results in accuracy reduction of $\mathrm{NI}+\mathrm{fBm}$ technique. On the contrary, within $\mathrm{NI}+\mathrm{DCT}$ approach the Hurst exponent estimates are used only at NI map forming stage, and they do not directly influence the STD estimation stage.

Availability of CRLB $\hat{\sigma}_{\sigma_{n} \cdot \mathrm{NI}}^{2}$ allows presenting noise STD estimated in the normalized form as

$$
\hat{\sigma}_{\text {norm }}=\frac{\left(\hat{\sigma}_{c}-\sigma_{n 0}\right)}{\widehat{\sigma}_{\sigma_{n} \cdot \mathrm{NI}}} .
$$

Considering $\sigma_{\sigma_{n} \cdot \mathrm{NI}}^{2}$ as potential noise STD estimation accuracy, $\hat{\sigma}_{\text {norm }}$ for an efficient unbiased estimator should approach normal distribution with zero mean and unit variance. Then, it becomes possible to compare the considered estimators to the efficient one.

Figure 10 presents four empirical pdfs of $\hat{\sigma}_{\text {norm }}$ for the $\mathrm{NI}+\mathrm{fBm}$ and NI+DCT, BM3D and SBIQ estimators (color settings are as in Figure 9). These pdfs have been obtained for all images and all analyzed noise variances. It is seen that the pdf of $\hat{\sigma}_{\text {norm }}$ for NI+DCT estimator follows Gaussian-like distribution with the mean equal to 0.22 and STD equal to 3.16. The pdf of $\hat{\sigma}_{\text {norm }}$ for the BM3D $(\mathrm{NI}+\mathrm{fBm})$ estimator is nonsymmetrical with mode close to zero, mean equal 
$2.30(-2.60)$ and STD equal to 5.31 (6.29). The empirical pdf for the SBIQ method is also nonsymmetrical one with heavy right and left tails. Its mean equals to -2.24 and its STD is 39.25 (taking into account the outliers mentioned above).

We see that accuracy of all considered estimators is quite far from $\sigma_{\sigma_{n} \cdot \mathrm{NI}}^{2}$. Their statistical efficiency with respect to this bound can be expressed as

$$
e=100 \% \cdot \frac{N_{e}}{\sum_{i=1}^{N_{e}} \hat{\sigma}_{\text {norm } \cdot i}^{2}},
$$

where the sum for each estimator is calculated over all $N_{e}$ available estimates. For the proposed estimators, we obtain $e_{\text {NI_DCT }}=10.01 \%$ and $e_{\text {NI_fBm }}=2.17 \%$. One has $e_{\text {BM3D }}=$ $3.00 \%$ for the BM3D estimator and $e_{S B I Q}=0.07 \%$ for the SBIQ. These results show that the proposed NI+DCT estimator is by 3 times more efficient that the state-of-the-art BM3D estimator, although performance of STD estimators can be further improved considerably. Thus, there is a room for further improvement and research in the area of noise STD blind estimation.

\section{Conclusion}

In this paper, a novel approach to image noise STD estimation has been proposed. It is mainly based on iterative separation of the processed image into two areas: noiseinformative one that is able to provide information on noise STD and texture-informative area that allows estimating texture correlation structure or roughness. The $2 \mathrm{D} \mathrm{fBm}$ model has been used as the model for image texture.

Such separation provides several advantages. First, it allows solving two complementary problems: to obtain accurate texture parameters for noisy part of the image and accurate noise parameters for textural part of the image, thus making both texture and noise parameters available for the whole image. Second, using texture and noise parameters, the Fisher information that a single SW contains about noise STD (or CRLB $\sigma_{\sigma_{n}}^{2}$ on noise STD estimates) has been determined to refine current NI and TI maps.

The experiments on TID2008 database have shown that separation on NI and TI maps can be successfully carried out for real-life images. NI maps for these images may occupy from 1 to $80 \%$ of image area depending on their homogeneity. The relative area of the NI map increases with noise variance.

Availability of CRLB $\sigma_{\sigma_{n}}^{2}$ allows determining potential variance of additive noise STD estimation from the whole NI map. We have used this bound to compare the efficiency of our estimators to that of two state-of-the-art estimators: BM3D and SBIQ method. We found NI+DCT estimator to significantly outperform the BM3D and SBIQ estimators. At the same time, the designed NI+DCT estimator provides noise STD estimates with STD approximately 3 times larger than the estimated potential STD $\hat{\sigma}_{\sigma_{n}} \cdot \mathrm{NI}$. Thus, the design of more efficient noise STD estimator is challenging.

\section{References}

[1] M. Elad, Sparse and Redundant Representations. From Theory to Applications in Signal and Image Processing, Springer Science + Business Media, LLC, 2010.

[2] B. Vozel, S. K. Abramov, and K. Chehdi, "Blind determination of noise type for spaceborne and airborne remote sensing," in Multivariate Image Processing, C. Collet, J. Chanussot, and K. Chehdi, Eds., chapter 9, p. 464, Wiley-ISTE, 2009.

[3] F. D. Van Der Meer and S. M. Dejong, Imaging Spectrometry: Basic Principles and Prospective Applications, Kluwer Academic Publishers, Dodrecht, The Netherlands, 2001.

[4] S. Gao and T. D. Bui, "Image segmentation and selective smoothing by using Mumford-Shah model," IEEE Transactions on Image Processing, vol. 14, no. 10, pp. 1537-1549, 2005.

[5] A. de Santis and D. Iacoviello, "A region growing method for medical images segmentation," International Journal of Tomography and Statistics, vol. 13, no. 10, pp. 19-37, 2010.

[6] V. V. Lukin, S. K. Abramov, N. N. Ponomarenko et al., "Processing of images based on blind evaluation of noise type and characteristics," in Image and Signal Processing for Remote Sensing, vol. 7477 of Proceedings of SPIE, p. 12, Berlin, Germany, 2009.

[7] P. Chatterjee and P. Milanfar, "Fundamental limits of image denoising: are we there yet?" in Proceedings of the IEEE International Conference on Acoustics, Speech and Signal Processing (ICASSP '10), pp. 1358-1361, Dallas, Tex, USA, 2010.

[8] K. Dabov, A. Foi, V. Katkovnik, and K. Egiazarian, "Image denoising by sparse 3-D transform-domain collaborative filtering," IEEE Transactions on Image Processing, vol. 16, no. 8, pp. 2080-2095, 2007.

[9] B. Aiazzi, L. Alparone, A. Barducci et al., "Noise modelling and estimation of hyperspectral data from airborne imaging spectrometers," Annals of Geophysics, vol. 49, no. 1, pp. 1-9, 2006.

[10] V. Lukin, S. Abramov, M. Uss et al., "Testing of methods for blind estimation of noise variance on large image database," in Theoretical and Practical Aspects of Digital Signal Processing in Informational-Telecommunication Systems, V. I. Marchuk, Ed., South-Russian State University of Economics and service, Shakhty, Russia, 2009, http://k504.xai.edu.ua/ html/prepods/lukin/BookCh1.pdf.

[11] B. C. Gao, "An operational method for estimating signal to noise ratios from data acquired with imaging spectrometers," Remote Sensing of Environment, vol. 43, no. 1, pp. 23-33, 1993.

[12] R. E. Roger and J. F. Arnold, "Reliably estimating the noise in AVIRIS hyperspectral images," International Journal of Remote Sensing, vol. 17, no. 10, pp. 1951-1962, 1996.

[13] N. N. Ponomarenko, V. V. Lukin, M. S. Zriakhov, A. Kaarna, and J. Astola, "An automatic approach to lossy compression of AVIRIS images," in Proceedings of the International Geoscience and Remote Sensing Symposium (IGARSS), pp. 472-475, Barcelona, Spain, 2008.

[14] A. Danielyan and A. Foi, "Noise variance estimation in nonlocal transform domain," in Proceedings of the International Workshop on Local and Non-Local Approximation in Image Processing (LNLA '09), pp. 41-45, August 2009.

[15] L. Şendur and I. W. Selesnick, "Bivariate shrinkage with local variance estimation," IEEE Signal Processing Letters, vol. 9, no. 12, pp. 438-441, 2002.

[16] A. De Stefano, P. R. White, and W. B. Collis, "Training methods for image noise level estimation on wavelet components," EURASIP Journal on Applied Signal Processing, vol. 2004, no. 16, pp. 2400-2407, 2004. 
[17] J. S. Lee and K. Hoppel, "Noise modeling and estimation of remotely-sensed images," Proceedings of the International Geoscience and Remote Sensing Symposium (IGARSS '89), vol. 2, pp. 1005-1008, 1989.

[18] L. Alparone, M. Selva, L. Capobianco, S. Moretti, L. Chiarantini, and F. Butera, "Quality assessment of data products from a new generation airborne imaging spectrometer," in Proceedings of the IEEE International Geoscience and Remote Sensing Symposium (IGARSS '09), vol. 4, pp. IV422-IV425, July 2009.

[19] V. V. Lukin, S. K. Abramov, A. A. Zelensky, J. T. Astola, B. Vozel, and K. Chehdi, "Improved minimal inter-quantile distance method for blind estimation of noise variance in images," in Image and Signal Processing for Remote Sensing, vol. 6748 of Proceedings of SPIE, p. 12, Florence, Italy, 2007.

[20] V. V. Lukin, S. K. Abramov, B. Vozel, and K. Chehdi, "A method for blind automatic evaluation of noise variance in images based on bootstrap and myriad operations," in Image and Signal Processing for Remote Sensing, vol. 5982 of Proceedings of SPIE, pp. 299-310, Bruges, Belgium, 2005.

[21] C. Liu, R. Szeliski, S. B. Kang, C. L. Zitnick, and W. T. Freeman, "Automatic estimation and removal of noise from a single image," IEEE Transactions on Pattern Analysis and Machine Intelligence, vol. 30, no. 2, pp. 299-314, 2008.

[22] B. Xu and P. Gong, "Noise estimation in a noise-adjusted principal component transformation and hyperspectral image restoration," Canadian Journal of Remote Sensing, vol. 34, no. 3, pp. 271-286, 2008.

[23] L. Klaine, B. Vozel, and K. Chehdi, "Unsupervised variational classification through image multi-thresholding," in Proceedings of the 13th EUSIPCO Conference, p. 4, Antalya, Turkey, 2005.

[24] M. Wettle, V. E. Brando, and A. G. Dekker, "A methodology for retrieval of environmental noise equivalent spectra applied to four Hyperion scenes of the same tropical coral reef," Remote Sensing of Environment, vol. 93, no. 1-2, pp. 188-197, 2004.

[25] S. K. Abramov, V. V. Lukin, B. Vozel, K. Chehdi, and J. T. Astola, "Segmentation-based method for blind evaluation of noise variance in images," SPIE Journal of Applied Remote Sensing, vol. 2, no. 1, p. 15, 2008.

[26] L. R. Gao, B. Zhang, X. Zhang, W. J. Zhang, and Q. X. Tong, "A new operational method for estimating noise in hyperspectral images," IEEE Geoscience and Remote Sensing Letters, vol. 5, no. 1, pp. 83-87, 2008.

[27] C. Kervrann and J. Boulanger, "Local adaptivity to variable smoothness for exemplar-based image regularization and representation," International Journal of Computer Vision, vol. 79, no. 1, pp. 45-69, 2008.

[28] W. Sun, G. Xu, P. Gong, and S. Liang, "Fractal analysis of remotely sensed images: a review of methods and applications," International Journal of Remote Sensing, vol. 27, no. 22, pp. 4963-4990, 2006.

[29] B. Pesquet-Popescu and J. L. Véhel, "Stochastic fractal models for image processing," IEEE Signal Processing Magazine, vol. 19 , no. 5, pp. 48-62, 2002.

[30] B. B. Mandelbrot and J. W. Van Ness, "Fractional brownian motions, fractional noises and applications," SIAM Review, vol. 10, no. 4, pp. 422-437, 1968.

[31] K. Falconer, Fractal Geometry: Mathematical Foundations and Applications, John Wiley \& Sons, 2003.

[32] D. G. Luenberger, Linear and Nonlinear Programming, Addison-Wesley, Reading, Mass, USA, 2nd edition, 1984.
[33] N. N. Ponomarenko, V. V. Lukin, M. S. Zriakhov, A. Kaarna, and J. Astola, "An automatic approach to lossy compression of AVIRIS images," in Proceedings of the International Geoscience and Remote Sensing Symposium (IGARSS '08), pp. 472-475, Barcelona, Spain, 2008.

[34] N. Ponomarenko, V. Lukin, K. Egiazarian, J. Astola, M. Carli, and F. Battisti, "Color image database for evaluation of image quality metrics," in Proceedings of the IEEE 10th Workshop on Multimedia Signal Processing (MMSP '08), pp. 403-408, October 2008.

[35] V. Lukin, S. Abramov, M. Uss, B. Vozel, and K. Chehdi, "Performance analysis of segmentation-based method for blind evaluation of additive noise in images," in Proceedings of MSMW, p. 3, Kharkov, Ukraine, 2010.

[36] A. Foi, "Clipped noisy images: heteroskedastic modeling and practical denoising," Signal Processing, vol. 89, no. 12, pp. 2609-2629, 2009. 